Алгебра и анализ

Том. 16 (2004), вып. 2
St. Petersburg Math. J.

Vol. 16 (2005), No. 2, Pages 401-412

S 1061-0022(05)00856-3

Article electronically published on March 9, 2005

\title{
HARMONIC DIFFEOMORPHISMS OF MANIFOLDS
}

\author{
S. E. STEPANOV AND I. G. SHANDRA
}

\begin{abstract}
In spite of the abundance of publications on harmonic mappings of manifolds, at present there exists neither a theory of harmonic diffeomorphisms, nor a definition of infinitesimal harmonic transformation of a Riemannian manifold, to say nothing of the theory of groups of such transformations. In the paper, this gap is partially filled, and a new subject of investigations is announced.
\end{abstract}

\section{$\S 0$. INTRODUCTION}

The theory of harmonic mappings dates back to investigation of minimal surfaces by Weierstrass. However, only in the 1950s the theory acquired an independent status. From the early 1960s, it has been developing quickly. Publications on harmonic mappings have long been numbered by hundreds (see the surveys [1]-[3]).

Despite the abundance of publications on harmonic mappings of Riemannian manifolds, at present there exists neither a theory of harmonic diffeomorphisms, nor a definition of an infinitesimal harmonic transformation, i.e., of a local harmonic autodiffeomorphism of a Riemannian manifold, to say nothing of the theory of groups of such transformations. This is in sharp contrast with the theories of isometric, conformal, projective, and other types of mappings (see, e.g., [4]-[ $]$ ).

The present paper partially fills this gap and announces a new subject of investigations. Here we study harmonic diffeomorphisms and local groups of harmonic transformations of Riemannian, almost complex, and complex manifolds by methods of the classical tensor analysis on manifolds; we also use elements of the group representation theory and the Bochner techniques [9].

In $\S 1$, we present facts about harmonic mappings of Riemannian manifolds, find a necessary and sufficient condition for a diffeomorphism to be harmonic, prove our main theorem on harmonic diffeomorphisms, and present examples of harmonic diffeomorphisms between Riemannian and almost complex manifolds.

In $\S 2$, we indicate two methods of classification of harmonic diffeomorphisms between Riemannian manifolds, which are based on the group representation theory; we describe one of them in detail and study three of seven distinguished classes of harmonic diffeomorphisms.

In $\S 3$, we define infinitesimal harmonic transformations, describe properties of the local one-parameter group of infinitesimal harmonic transformations of Riemannian manifolds, and present examples of infinitesimal harmonic transformations of Riemannian and Kähler manifolds.

The results presented in the paper were announced in a plenary talk at an international conference 10 .

2000 Mathematics Subject Classification. Primary 53C43, 58E20.

Key words and phrases. Riemannian manifold, harmonic diffeomorphism, infinitesimal harmonic transformation. 


\section{$\S 1$. HARMONIC MAPPINGS OF RiEMANNIAN MANIFOLDS}

1.1. Preliminaries. We consider Riemannian $C^{\infty}$-manifolds $(M, g)$ and $(\bar{M}, \bar{g})$ of dimensions $m$ and $n$, respectively, and a smooth mapping $f: M \rightarrow \bar{M}$. The differential $f_{*}: T M \rightarrow T \bar{M}$ of $f$ is a section $d f$ of the tensor vector bundle $T^{*} M \otimes f^{-1}(T \bar{M})$, where $f^{-1}(T \bar{M})_{x}=T_{f(x)} \bar{M}$ at each point $x \in M$.

For a relatively compact open subset $\Omega \subset M$, the energy $E_{\Omega}(f)$ of the mapping $f$ is the functional

$$
E_{\Omega}(f)=\frac{1}{2} \int_{\Omega}\|d f\|^{2} d V,
$$

where $\|d f\|$ is the norm of $d f$ calculated in the natural Riemannian metric on $T^{*} M \otimes$ $f^{-1}(T \bar{M})$, and $d V$ is the element of volume of $(M, g)$ (see, e.g., [3]).

Definition 1.1. A smooth mapping $f:(M, g) \rightarrow(\bar{M}, \bar{g})$ is said to be harmonic if $f$ provides an extremum of the energy functional $E_{\Omega}(f)$ for each relatively compact open subset $\Omega \subset M$ with respect to the variations of $f$ that are compactly supported in $\Omega$.

The Levi-Civita connections $\nabla$ and $\bar{\nabla}$ of $(M, g)$ and $(\bar{M}, \bar{g})$ induce a connection $D$ in the vector bundle $T^{*} M \otimes f^{-1}(T \bar{M})$. We have the following result.

Theorem $1.1[1]-3]$. A smooth mapping $f:(M, g) \rightarrow(\bar{M}, \bar{g})$ is harmonic if and only if it satisfies the Euler-Lagrange equation

$$
\operatorname{trace}_{g} D(d f)=0 .
$$

We write the Euler-Lagrange equations in local coordinates. Suppose $\left\{x^{1}, \ldots, x^{m}\right\}$ are local coordinates in a neighborhood $U$ of a point $x \in M$ and $\left\{\bar{x}^{1}, \ldots, \bar{x}^{n}\right\}$ are local coordinates in a neighborhood $\bar{U} \supset f(U)$ of the point $\bar{x}=f(x) \in \bar{M}$. In what follows, we denote by $\Gamma_{i j}^{k}, i, j, k, \ldots=1,2, \ldots, m$, and by $\bar{\Gamma}_{\beta \gamma}^{\alpha}, \alpha, \beta, \gamma, \ldots=1,2, \ldots, n$, the Christoffel symbols of the Levi-Civita connections $\nabla$ and $\bar{\nabla}$, respectively. Then the Euler-Lagrange equations (1.1) take the form

$$
g^{i j}\left(\partial_{i} \partial_{j} f^{a}-\Gamma_{i j}^{k} \partial_{k} f^{a}+\bar{\Gamma}_{\beta, \gamma}^{a} \partial_{i} f^{\beta} \partial_{j} f^{\gamma}\right)=\Delta f^{a}+g^{i j} \bar{\Gamma}_{\beta, \gamma}^{a} \partial_{i} f^{\beta} \partial_{j} f^{\gamma}=0,
$$

where the $g^{i j}$ are the contravariant components of the metric tensor $g, \partial_{i}=\partial / \partial x^{i}$, and $\Delta$ is the Laplace-Beltrami operator of $(M, g)$ (see, e.g., 3, 12]).

Remark. In the case of Euclidean space, equations (1.2) turn into the system of LaplaceBeltrami equations $\Delta f^{a}=\sum_{j=1}^{m} \partial_{j}^{2} f^{a}=0$, whence the term "harmonic mapping".

Suppose that $\operatorname{dim} M=\operatorname{dim} \bar{M}=m$ and $f: M \rightarrow \bar{M}$ is a diffeomorphism. Then, locally, the mapping $f$ acts in accordance with the "equality of coordinates" rule $\bar{x}^{1}=$ $x^{1}, \ldots, \bar{x}^{m}=x^{m}$ for the corresponding points $x$ and $\bar{x}=f(x)$ (see [13, p. 67]). In this case, we say that $\left\{x^{1}, \ldots, x^{m}\right\}$ are $f$-adjusted common (local) coordinates on $M$ and $\bar{M}$ (see [6, p. 47]).

We denote by $g_{i j}$ and $\bar{g}_{i j}$ the components of the metric tensors $g$ and $\bar{g}$, and by $\Gamma_{i j}^{k}$ and $\bar{\Gamma}_{i j}^{k}$ the Christoffel symbols of the Levi-Civita connections $\nabla$ and $\bar{\nabla}$ of $(M, g)$ and $(\bar{M}, \bar{g})$ in $f$-adjusted common coordinates $\left\{x^{1}, \ldots, x^{m}\right\}$. Then the Euler-Lagrange equations (1.2) take the form

$$
g^{i j} T_{i j}^{k}=0
$$

where $T_{i j}^{k}=\bar{\Gamma}_{i j}^{k}-\Gamma_{i j}^{k}$ are the components of the deformation tensor $T=\bar{\nabla}-\nabla$ of the connection $\nabla$ under the mapping $f$ (see [6, p. 71]). Conditions (1.3) mean that the deformation tensor $T$ is a symmetric traceless tensor field, i.e., a section of the tensor vector bundle $T M \otimes S_{0}^{2} M$. Thus, we have proved the following result. 
Corollary 1.2. Suppose $(M, g)$ and $(\bar{M}, \bar{g})$ are Riemannian manifolds with Levi-Civita connections $\nabla$ and $\bar{\nabla}$, respectively. A diffeomorphism $f: M \rightarrow \bar{M}$ is harmonic if and only if the deformation tensor $T=\bar{\nabla}-\nabla$ of $\nabla$ under the mapping $f$ is a section of the tensor vector bundle $T M \otimes S_{0}^{2} M$.

1.2. Examples of harmonic diffeomorphisms $f:(M, g) \rightarrow(\bar{M}, \bar{g})$.

1.2.1. Conformal diffeomorphisms. Our first example is a conformal diffeomorphism, which is characterized by the equality $\bar{g}=e^{2 \sigma} g$ in adjusted common coordinates $\left\{x^{1}, \ldots\right.$, $\left.x^{m}\right\}$. This equality implies the following relation between the Christoffel symbols (see [4. p. 113]):

$$
\bar{\Gamma}_{i j}^{k}=\Gamma_{i j}^{k}+\sigma_{i} \delta_{j}^{k}+\sigma_{j} \delta_{i}^{k}-\sigma^{k} g_{i j},
$$

where $\sigma_{i}=\partial_{i} \sigma, \sigma^{k}=g^{k i} \sigma_{i}$ and $\delta_{i}^{k}$ is the Kronecker delta. Obviously, the Euler-Lagrange equations (1.3) are fulfilled for a conformal diffeomorphism only in one of the following two cases: either $m=2$, or $\sigma=$ const.

Summing up, we see that a conformal diffeomorphism $f$ between two Riemannian 2-manifolds is always a harmonic mapping (cf. 3, p. 13]). For other dimensions, this is possible only if the diffeomorphism $f$ is a homothety.

1.2.2. As the second example of a harmonic diffeomorphism, we consider the composition of a diffeomorphism and a projective diffeomorphism.

Theorem 1.3. Suppose $(M, g),(\bar{M}, \bar{g})$, and $(\widetilde{M}, \tilde{g})$ are Riemannian m-manifolds $(m \geq$ $3), f: M \rightarrow \bar{M}$ is a conformal diffeomorphism, and $\bar{f}: \bar{M} \rightarrow \widetilde{M}$ is a projective diffeomorphism. The composition $\bar{f} \circ f: M \rightarrow \widetilde{M}$ is a harmonic mapping if and only if for each function

$$
\sigma=\frac{\ln (\operatorname{det} \tilde{g} / \operatorname{det} g)}{m^{2}+m-2}+\text { const }
$$

we have $\bar{g}=e^{2 \sigma} g$.

Proof. We recall that, by definition, a projective diffeomorphism $\bar{f}: \bar{M} \rightarrow \widetilde{M}$ maps the geodesics of $(\bar{M}, \bar{g})$ to those of $(\widetilde{M}, \tilde{g})$. In this case, in $\bar{f}$-adjusted common coordinates the Christoffel symbols $\widetilde{\Gamma}_{i j}^{k}$ and $\bar{\Gamma}_{i j}^{k}$ of $(\widetilde{M}, \tilde{g})$ and $(\bar{M}, \bar{g})$, respectively, satisfy the relation

$$
\widetilde{\Gamma}_{i j}^{k}=\bar{\Gamma}_{i j}^{k}+\psi_{i} \delta_{j}^{k}+\psi_{j} \delta_{i}^{k}
$$

where

$$
\psi_{j}=\frac{\partial_{j} \ln (\operatorname{det} \tilde{g} / \operatorname{det} \bar{g})}{2(m+1)}
$$

(see [4, pp. 70-76]) and [6] pp. 161-166]).

Now we consider the composition $\bar{f} \circ f: M \rightarrow \widetilde{M}$ indicated in the statement of the theorem. From (1.4) and (1.5) it follows that in $(\tilde{f} \circ f)$-adjusted common coordinates we have the relation

$$
\widetilde{\Gamma}_{i j}^{k}=\Gamma_{i j}^{k}+\left(\sigma_{i}+\psi_{i}\right) \delta_{j}^{k}+\left(\sigma_{j}+\psi_{j}\right) \delta_{i}^{k}-\sigma^{k} g_{i j} .
$$

Therefore, the Euler-Lagrange equations (1.3) for the mapping $\bar{f} \circ f$ take the form $d[(m-2) \sigma-2 \psi]=0$. For $m \geq 3$, this implies the required expression for the function $\sigma$. 
1.2.3. Now we consider harmonic diffeomorphisms of almost Hermitian manifolds. We recall (see [14, p. 139]) that an almost Hermitian manifold $(M, g, J)$ is an even-dimensional $C^{\infty}$-manifold $M$ endowed with an almost complex structure $J$ and a Riemannian metric $g$ with the following properties: at each point $x \in M, J$ is an endomorphism of the tangent space $T_{x} M$ such that $J^{2}=-\mathrm{id}$, and $g$ is invariant with respect to $J$, i.e., $g(J, J)=g$.

On an almost Hermitian manifold, the fundamental form $\Omega$ with local components $\Omega_{i j}=g_{i k} J_{j}^{k}$ is defined in a natural way (see [14 p. 140]). If the fundamental form $\Omega$ is coclosed, then the almost Hermitian manifold $(M, g, J)$ is said to be almost semi-Kähler (see, e.g., [15, 16]).

We mention three subclasses of this class of manifolds. The first is the class of almost Kähler manifolds, which are characterized by the requirement that the fundamental form $\Omega$ be closed, i.e., $d \Omega=0$ (see [14, p. 141]). The second class is that of nearly Kähler manifolds (see [15, 17]), for which the fundamental form $\Omega$ is a Killing form [20, p. 339], i.e., $\nabla \Omega=d \Omega$. Finally, the third class consists of Kähler manifolds with $\nabla \Omega=0$ (see [14, p. 141]).

A mapping $f: M \rightarrow \bar{M}$ of an almostHermitian manifold $(M, g, J)$ to an almost Hermitian manifold $(\bar{M}, \bar{g}, \bar{J})$ is almost complex if $f_{*} \circ J=\bar{J} \circ f_{*}$ (see [14, p. 118]).

Theorem 1.4. Suppose $(M, g, J)$ is an almost Hermitian manifold and $(\bar{M}, \bar{g}, \bar{J})$ is a nearly Kähler manifold. Then an almost complex diffeomorphism $f: M \rightarrow \bar{M}$ is a harmonic mapping if and only if the manifold $(M, g, J)$ is almost semi-Kähler.

Proof. In $f$-adjusted common coordinates, we have $J_{j}^{i}=\bar{J}_{j}^{i}$. Differentiating these relations covariantly, we obtain

$$
\nabla_{k} J_{j}^{i}=\bar{\nabla}_{k} \bar{J}_{j}^{i}+\bar{J}_{l}^{i} T_{j k}^{l}-\bar{J}_{j}^{l} T_{l k}^{i} .
$$

Convolution of both sides in (1.7) with $g^{k j}$ yields

$$
\nabla_{k} J^{i k}=\frac{1}{2} g^{k j}\left(\bar{\nabla}_{k} \bar{J}_{j}^{i}+\bar{\nabla}_{j} \bar{J}_{k}^{i}\right)+\bar{J}_{i}^{i}\left(g^{j k} T_{j k}^{i}\right) .
$$

Since $(\bar{M}, \bar{g}, \bar{J})$ is nearly Kähler, these equations take the form

$$
\nabla_{k} J^{i k}=\bar{J}_{i}^{i}\left(g^{j k} T_{j k}^{i}\right) \text {. }
$$

Now the claim is obvious.

Corollary 1.5. If $(M, g, J)$ and $(\bar{M}, \bar{g}, \bar{J})$ are two nearly Kähler manifolds, then any almost complex diffeomorphism $f: M \rightarrow \bar{M}$ is a harmonic mapping.

Remark. For Kähler manifolds, an almost complex mapping is holomorphic (see [14 p. 118]). Therefore, Theorem 1.4 implies a known result saying that a holomorphic diffeomorphism between two Kähler manifolds is a harmonic mapping.

\subsection{A criterion for a diffeomorphism to be harmonic.}

Theorem 1.6. Suppose $(M, g)$ and $(\bar{M}, \bar{g})$ are Riemannian manifolds with Levi-Civita connections $\nabla$ and $\bar{\nabla}$, respectively. Then a diffeomorphism $f: M \rightarrow \bar{M}$ is harmonic if and only if the differential equations $\nabla^{k} \bar{g}_{k j}=\frac{1}{2} \nabla_{j}\left(g^{i l} \bar{g}_{i l}\right)$ for $\nabla^{k}=g^{k j} \nabla_{j}$ or the differential equations $g^{k l} \bar{\nabla}_{k} g_{l j}=\frac{1}{2} g^{k l} \bar{\nabla}_{j} g_{k l}$ are fulfilled in $f$-adjusted common coordinates.

Proof. Observing that

$$
\begin{aligned}
& \bar{\nabla}_{k} g_{i j}=-g_{l j} T_{i k}^{l}-g_{i l} T_{j k}^{l}, \\
& \nabla_{k} \bar{g}_{i j}=\bar{g}_{l j} T_{i k}^{l}+\bar{g}_{i l} T_{j k}^{l},
\end{aligned}
$$


we find the following expressions for the deformation tensor:

$$
\begin{aligned}
T_{i j}^{k} & =g^{k l}\left(-\bar{\nabla}_{i} g_{j l}-\bar{\nabla}_{j} g_{i l}+\bar{\nabla}_{l} g_{i j}\right), \\
T_{i j}^{k} & =\bar{g}^{k l}\left(\nabla_{i} \bar{g}_{j l}+\nabla_{j} \bar{g}_{i l}-\nabla_{l} \bar{g}_{i j}\right),
\end{aligned}
$$

where the $\bar{g}^{k l}$ are the components of the matrix inverse to that of the metric tensor $\bar{g}$. Then, using (1.9), we can transform the Euler-Lagrange equations (1.3) to $g^{k l} \bar{\nabla}_{k} g_{l j}=$ $\frac{1}{2} g^{k l} \bar{\nabla}_{j} g_{k l}$, or to

$$
\nabla^{k} \bar{g}_{k j}=\frac{1}{2} \nabla_{j}\left(g^{i l} \bar{g}_{i l}\right)
$$

\subsection{Vanishing theorem.}

Theorem 1.7. Suppose $(M, g)$ is a complete Riemannian manifold with positive Ricci curvature and $(\bar{M}, \bar{g})$ is a Riemannian manifold with nonpositive sectional curvature. Then there exist no harmonic diffeomorphisms $M \rightarrow \bar{M}$.

Proof. We fix $f$-adjusted common coordinates on $(\bar{M}, \bar{g})$ and $(M, g)$. Starting with the components $\bar{R}_{i j k l}$ of the curvature tensor $\bar{R}$ of $(\bar{M}, \bar{g})$ and the components $R_{i j}$ of the Ricci tensor Ric of $(M, g)$, we construct two scalar invariants: $r=g^{i k} g^{i l} \bar{R}_{i j k l}$ and $s=\bar{g}^{i j} R_{i j}$.

A vanishing theorem for harmonic mappings of Riemannian manifolds of arbitrary dimensions was proved in 9]. By that theorem, a diffeomorphism $f: M \rightarrow \bar{M}$ of a compact oriented Riemannian manifold $(M, g)$ to a Riemannian manifold $(\bar{M}, \bar{g})$ is not harmonic if $r(f)<s(f)$, where

$$
r(f)=\int_{M} g^{i k} g^{i l} \bar{R}_{i j k l} \text { Vol and } \quad s(f)=\int_{M} \bar{g}^{i j} R_{i j} \text { Vol } .
$$

Obviously, this inequality is fulfilled automatically if the sectional curvature of $(\bar{M}, \bar{g})$ is nonpositive; on the contrary, the Ricci curvature of $(M, g)$ is everywhere positive.

Remark. The theorem in [9] mentioned above was proved with the help of the Green theorem [23. p. 259]; to apply it we must assume that $(M, g)$ is compact and oriented. Since a complete Riemannian manifold with positive Ricci curvature is compact 11 p. 117], the compactness assumption in Theorem 1.6 is replaced by the assumption of completeness of $(M, g)$. Furthermore, the assumption of orientability of $(M, g)$ in the same theorem is also immaterial because if $M$ is nonorientable, then we can consider the double cover of $M$ which is orientable (see, e.g., 8, p. 308] and [14, p. 63]).

\section{§2. SeVen Classes of harmonic Diffeomorphisms}

2.0. By Theorem 1.6, the components $G_{i j}$ of the tensor field $G=\bar{g}-\frac{1}{2}\left(\operatorname{trace}_{g} \bar{g}\right) g$ satisfy the differential equations $\nabla^{k} G_{k j}=0$ if and only if the diffeomorphism $f: M \rightarrow \bar{M}$ is harmonic.

We consider the space

$$
\mathcal{G}(E)=\left\{\widetilde{G} \in E^{*} \otimes S^{2} E \mid \widetilde{G}_{12}(c)=0\right\},
$$

where $E$ is an $m$-dimensional Euclidean vector space and $\widetilde{G}_{23}(c)=\sum_{k=1}^{m} \widetilde{G}\left(c, e_{k}, e_{k}\right)$ for an orthonormal basis $\left\{e_{1}, \ldots, e_{m}\right\}$ and an arbitrary $c$ in $E$. By [18], the tensor space $\mathcal{G}(E)$ is the sum of three subspaces irreducible with respect to the action of the orthogonal 


$$
\begin{aligned}
& \operatorname{group} O(m, \mathbb{R}) \text { : } \\
& \qquad \begin{aligned}
\mathcal{G}_{1}(E)=\{\widetilde{G} \in \mathcal{G}(E) \mid \widetilde{G}(a, b, c)=\widetilde{G}(b, a, c)\} \\
\mathcal{G}_{2}(E)=\{\widetilde{G} \in \mathcal{G}(E) \mid \widetilde{G}(a, b, c)+\widetilde{G}(b, c, a)+\widetilde{G}(c, a, b)=0\}, \\
\mathcal{G}_{3}(E)=\left\{\widetilde{G} \in \mathcal{G}(E) \mid \widetilde{G}(a, b, c)=\left(m^{2}+m-2\right)^{-1}\right. \\
\left.\quad \times\left\lfloor(m+1) \widetilde{G}_{23}(a) q(b, c)-\widetilde{G}_{23}(b) q(a, c)-\widetilde{G}_{23}(c) q(a, b)\right\rfloor\right\} .
\end{aligned}
\end{aligned}
$$

The tensor field $\nabla G$ on $(M, g)$ is a section of the vector bundle $\mathcal{G}(T M)$, the fiber of which at each point is the space $\mathcal{G}(E)$. As a consequence, we obtain a pointwise decomposition of $\nabla G$ into a sum of the tensor fields corresponding to the pointwise irreducible components of the action of the group $O(m, \mathbb{R})$. This decomposition of $\nabla G$ determines a "coarse" classification of harmonic diffeomorphisms, where each class consists of diffeomorphisms for which $\nabla G$ is a section of one of the invariant subbundles $\mathcal{G}_{1}(T M)$, $\mathcal{G}_{2}(T M)$, and $\mathcal{G}_{3}(T M)$ or of their direct sums.

Definition 2.1. Let $(M, g)$ and $(\bar{M}, \bar{g})$ be Riemannian manifolds with Levi-Civita connections $\nabla$ and $\bar{\nabla}$, respectively, and let $G=\bar{g}-\frac{1}{2}\left(\operatorname{trace}_{g} \bar{g}\right) g$. A harmonic diffeomorphism $f: M \rightarrow \bar{M}$ belongs to the class $\mathcal{I}$ if at each point $x \in M$ the tensor field $\nabla G$ belongs to the subspace $\mathcal{I}\left(T_{x} M\right)$ of the tensor space $\mathcal{G}\left(T_{x} M\right)$.

We complete the list of classes with yet another class for which $\nabla G$ is a section of the subbundle $\mathcal{G}_{1}(T M) \cap \mathcal{G}_{2}(T M) \cap \mathcal{G}_{3}(T M)$, i.e., $\nabla G=0$.

Theorem 2.1. Suppose $(M, g)$ and $(\bar{M}, \bar{g})$ are two Riemannian manifolds. In an invariant way, we can distinguish seven classes of harmonic diffeomorphisms $f: M \rightarrow \bar{M}$ for each of which the field $\nabla G$, where $G=\bar{g}-\frac{1}{2}\left(\right.$ trace $\left._{g} \bar{g}\right) g$, is a section of the corresponding invariant subbundle $\mathcal{G}_{1}(T M), \mathcal{G}_{2}(T M), \mathcal{G}_{3}(T M)$, or $\mathcal{G}_{1}(T M) \cap \mathcal{G}_{2}(T M) \cap \mathcal{G}_{3}(T M)$, or of one of their direct sums.

We consider the first three classes of harmonic diffeomorphisms.

2.1. Class $\mathcal{I}_{1}$. Suppose $f \in \mathcal{I}_{1}$. This means that the components of the tensor field $\bar{g}$ satisfy equations (1.10) and also satisfy the differential equations

$$
\nabla_{k} \bar{g}_{i j}-\frac{1}{2} g_{i j} \nabla_{k}\left(g^{n l} \bar{g}_{n l}\right)=\nabla_{i} \bar{g}_{k j}-\frac{1}{2} g_{k j} \nabla_{i}\left(g^{n l} \bar{g}_{n l}\right) .
$$

The collection of these equations is equivalent to the system of differential equations

$$
\begin{aligned}
\nabla_{k}\left(g^{n l} \bar{g}_{n l}\right) & =0, \\
\nabla_{k} \bar{g}_{i j} & =\nabla_{i} \bar{g}_{k j} .
\end{aligned}
$$

From (2.2) it follows that on $(M, g)$ the tensor field $\bar{g}$ is a Codazzi tensor with constant trace. The geometry of manifolds bearing Codazzi tensor fields is described in detail in the literature (see the survey in [19, pp. 590-598]). For this reason, the facts established earlier can be used for a description of the geometry of harmonic diffeomorphisms of class $\mathcal{I}_{1}$. For example, we have the following result.

Theorem 2.3. Suppose $(M, g)$ and $(\bar{M}, \bar{g})$ are two Riemannian manifolds, and $(M, g)$ has constant sectional curvature $K$. A diffeomorphism $f: M \rightarrow \bar{M}$ is harmonic of class $\mathcal{I}_{1}$ if and only if the tensor $\bar{g}$ has the form

$$
\bar{g}=\operatorname{Hess}(F)+K F g,
$$

where the function $F$ on $(M, g)$ is a solution of the Poisson equation $\Delta F+n K F=C$ for a constant $C>0$. 
Proof. On the one hand, $f$ is harmonic of class $\mathcal{I}_{1}$ if and only if equations (2.1) and (2.2) are fulfilled. On the other hand (see [19, p. 591]), since $(M, g)$ has constant sectional curvature $K$, the general solution of (2.2) has the form $\bar{g}=\operatorname{Hess}(F)+K F g$, where $F$ is an arbitrary differentiable function on $(M, g)$ and $\operatorname{Hess}(F)$ is the Hessian of $F$, $\operatorname{Hess}(F)=\nabla(d F)$ (see [19 p. 53]). In this case, equations (2.1) take the form of the Poisson equation $\Delta F+n K F=C$ for some constant $C>0$. Finally, observe that we must look only for the solutions $F$ of the Poisson equation that ensure the positive definiteness of the field $\bar{g}$. For example, in the trivial case where $f$ is a homothety of a manifold $(M, g)$ of constant sectional curvature $K>0$, we set $F=K^{-1} e^{2 \sigma}$ because $\bar{g}=e^{2 \sigma} g$ for $\sigma=$ const.

Applying M. Berger's theorem [7, p. 591] on the Killing tensors with constant trace, we arrive at the following result.

Theorem 2.4. Suppose $(M, g)$ is a compact orientable Riemannian manifold of nonnegative sectional curvature $K$, and $K>0$ for at least one point. Then each harmonic diffeomorphism $f \in \mathcal{I}_{1}$ of $(M, g)$ to another Riemannian manifold $(\bar{M}, \bar{g})$ is a homothety.

2.2. Class $\mathcal{I}_{2}$. Now suppose that $f \in \mathcal{I}_{2}$. This means that the components of the tensor field $\bar{g}$ satisfy (1.10) and the differential equations

$\left[\nabla_{k} \bar{g}_{i j}-\frac{1}{2} g_{i j} \nabla_{k}\left(g^{n l} \bar{g}_{n l}\right)\right]+\left[\nabla_{i} \bar{g}_{k j}-\frac{1}{2} g_{k j} \nabla_{i}\left(g^{n l} \bar{g}_{n l}\right)\right]+\left[\nabla_{j} \bar{g}_{k i}-\frac{1}{2} g_{k i} \nabla_{j}\left(g^{n l} \bar{g}_{n l}\right)\right]=0$.

For $m \geq 3$, the system of all these equations is equivalent to the following system of differential equations:

$$
\begin{aligned}
\nabla_{k}\left(g^{n l} \bar{g}_{n l}\right) & =0, \\
\nabla_{k} \bar{g}_{i j}+\nabla_{i} \bar{g}_{k j}+\nabla_{i} \bar{g}_{k j} & =0 .
\end{aligned}
$$

The form of equations (2.4) implies that on $(M, g)$ the tensor field $\bar{g}$ is a Killing tensor with constant trace (see [20, pp. 339-340]).

The geometry of manifolds bearing Killing tensor fields is described in detail in the literature. We can apply the known facts for describing the geometry of harmonic diffeomorphisms of class $\mathcal{I}_{2}$. For example, a Killing tensor field on a Riemannian manifold determines the first quadratic integral of the equations of geodesics (see 44 pp. 157-161]). Therefore, the existence of quadratic integrals of the equations of geodesics is a necessary condition for the existence of a harmonic diffeomorphism $f: M \rightarrow \bar{M}$ of class $\mathcal{I}_{2}$.

Theorem 2.5. Suppose $(M, g)$ and $(\bar{M}, \bar{g})$ are two Riemannian manifolds. If $(M, g)$ is locally flat, then a diffeomorphism $f: M \rightarrow \bar{M}$ is harmonic of class $\mathcal{I}_{2}$ if and only if in $f$-adjusted common coordinates $\left\{x^{1}, \ldots, x^{m}\right\}$ the tensor $\bar{g}$ has the form

$$
\bar{g}_{i j}=A_{i j k l} x^{k} x^{l}+B_{i j k} x^{k}+C_{i j}
$$

for some constants $A_{i j k l}, B_{i j k}, C_{i j}$ that are symmetric with respect to the first two subscripts and such that

$$
\begin{gathered}
A_{i j k l}+A_{j k i l}+A_{k i j l}=0, \\
B_{i j k}+B_{j k i}+B_{k i j}=0, \\
g^{i j} A_{i j k l}=g^{i j} B_{i j k}=0 .
\end{gathered}
$$

Proof. On the one hand, $f$ is harmonic of class $\mathcal{I}_{2}$ if and only if equations (2.3) and (2.4) are fulfilled. On the other hand (see [21]), since $(M, g)$ is a locally flat Riemannian manifold, the general solution of (2.4) has the form (2.5) for constants $A_{i j k l}, B_{i j k}$, and $C_{i j}$ symmetric with respect to the first two subscripts and satisfying (2.6). In this case, 
equations (2.3) take the equivalent form (2.7). Finally, we observe that, besides the conditions (2.6) and (2.7), the choice of the constants $A_{i j k l}, B_{i j k}$, and $C_{i j}$ in (2.5) is restricted by the condition of positive definiteness of the tensor field $\bar{g}$.

Suppose that $(M, g)$ is a compact orientable Riemannian manifold with $K \leq 0$ and that $K<0$ for at least one point. In [22] it was established that each Killing tensor field on $M$, and in particular the Killing field $\bar{g}$, has the form $\bar{g}=C g$, where $C=$ const. This implies the following result.

Theorem 2.6. Suppose $(M, g)$ is a compact orientable Riemannian manifold of nonpositive sectional curvature $K$, and $K<0$ for at least one point. Then each harmonic diffeomorphism $f \in \mathcal{I}_{2}$ of $(M, g)$ to another Riemannian manifold $(\bar{M}, \bar{g})$ is a homothety.

2.3. Class $\mathcal{I}_{3}$. The third class $\mathcal{I}_{3}$ of harmonic diffeomorphisms is related to the following system of differential equations:

$$
\nabla_{k} \bar{g}_{i j}=\frac{m g_{i j} \nabla_{k} \bar{g}_{l}^{l}+\frac{m-2}{2} g_{k j} \nabla_{i} \bar{g}_{l}^{l}+\frac{m-2}{2} g_{k i} \nabla_{j} \bar{g}_{l}^{l}}{(m+2)(m-1)} .
$$

We introduce the symmetric tensor field $\varphi$ with components

$$
\varphi_{i j}=\bar{g}_{i j}-\frac{m}{(m+2)(m-1)}\left(g^{k l} \bar{g}_{k l}\right) g_{i j}
$$

which, as easily follows from (2.8), satisfy the differential equations

$$
2 \nabla_{k} \varphi_{i j}=\left(g^{n l} \nabla_{i} \varphi_{n l}\right) g_{k j}+\left(g^{n l} \nabla_{j} \varphi_{n l}\right) g_{k i}
$$

By N. S. Sinyukov's theorem (see [6, p. 122]), a Riemannian manifold $(M, g)$ bears a symmetric tensor field $\varphi$ with components satisfying $(2.9)$ if and only if $(M, g)$ admits a projective diffeomorphism to some (pseudo-)Riemannian manifold $(\widetilde{M}, \tilde{g})$.

Conversely, suppose that a Riemannian manifold $(M, g)$ admits a projective diffeomorphism, and therefore there exists a symmetric tensor field $\varphi$ on $(M, g)$ the components of which satisfy equations (2.9).

The proof of the Sinyukov theorem implies that if $(\widetilde{M}, \tilde{g})$ is a Riemannian (rather than a pseudo-Riemannian) manifold, then the tensor field $\varphi$ must be positive definite. For this reason, we assume that $\varphi$ is positive definite and consider the tensor field $\bar{g}$ on $(M, g)$ with components $\bar{g}_{i j}=\varphi_{i j}+\frac{m}{m-2}\left(g^{k i} \varphi_{k l}\right) g_{i j}$. We can check that these components satisfy (2.8). Therefore, the diffeomorphism id $:(M, g) \rightarrow(M, \bar{g})$ is harmonic of class $\mathcal{I}_{3}$. Thus, we have proved the following result.

Theorem 2.7. Suppose $(M, g)$ is a Riemannian manifold. If $(M, g)$ admits a harmonic diffeomorphism $f \in \mathcal{I}_{3}$ to a Riemannian manifold $(\bar{M}, \bar{g})$, then $(M, g)$ also admits a projective diffeomorphism to some (pseudo-)Riemannian manifold $(\widetilde{M}, \bar{g})$. Furthermore, if $(M, g)$ admits a projective diffeomorphism to a Riemannian manifold $(\widetilde{M}, \tilde{g})$, then $(M, g)$ also admits a harmonic diffeomorphism $f \in \mathcal{I}_{3}$ to some Riemannian manifold $(\bar{M}, \bar{g})$.

We note that the theory of projective diffeomorphisms is well developed (see [6]), and hence, when studying the geometry of harmonic diffeomorphisms of the third class, we can use the known facts. For example, we prove the following result.

Corollary 2.8. Suppose $\left(M_{1}, g_{1}\right)$ is a Riemannian 1-manifold and $\left(M_{2}, g_{2}\right)$ is a Riemannian $(m-1)$-manifold. Then the cross-product $M_{1} \times_{F} M_{2}$ admits a harmonic diffeomorphism $f \in \mathcal{I}_{3}$ to some Riemannian m-manifold $(\bar{M}, \bar{g})$. 
Proof. We recall that the cross-product $M_{1} \times_{F} M_{2}$ of two Riemannian manifolds $\left(M_{1}, g_{1}\right)$ and $\left(M_{2}, g_{2}\right)$ is a manifold of the form $(M, g)=\left(M_{1} \times M_{2}, g_{1} \oplus F g_{2}\right)$ for a positive $F \in C^{\infty} M_{1}$ (see [19, p. 328]). It is known that if $\operatorname{dim} M_{1}=1$, then $M_{1} \times_{F} M_{2}$ admits a projective diffeomorphism to some Riemannian manifold $(\bar{M}, \bar{g})$ (see [6, pp. 98, 123]). Applying Theorem 2.7, we obtain the assertion.

For harmonic diffeomorphisms $f \in \mathcal{I}_{3}$, we have the following statement, which completely repeats Theorem 2.6.

Theorem 2.9. Suppose that $(M, g)$ is a compact orientable Riemannian manifold of nonpositive sectional curvature $K$ and that $K<0$ for at least one point. Then each harmonic diffeomorphism $f \in \mathcal{I}_{3}$ of $(M, g)$ to another Riemannian manifold $(\bar{M}, \bar{g})$ is a homothety.

Proof. We introduce the tensor field $\psi$ with local components $\psi_{i j}=\varphi_{i j}-\left(g^{k l} \varphi_{k l}\right) g_{i j}=$ $\bar{g}_{i j}-2(m+2)^{-1}\left(g^{k l} \bar{g}_{k l}\right) g_{i j}$. By (2.8) and (2.9), the field $\psi$ is Killing. Therefore, on a compact orientable manifold $(M, g)$ where $K \leq 0$ and at least at one point we have $K<0$, the field $\psi$ has the form $\psi=C g$, where $C=$ const (see [22]). The latter means that

whence

$$
g^{k l} \varphi_{k l}=\frac{m-2}{(m+2)(m-1)} g^{k l} \bar{g}_{k l}=\text { const },
$$

$$
\bar{g}=\bar{C} g, \quad \text { where } \bar{C}=\text { const. }
$$

Remark. There is another method for classification of harmonic diffeomorphisms (see [10]). In order to apply it, we must use the pointwise $O(m, \mathbb{R})$-irreducible decomposition of the space $T M \otimes S_{0}^{2} M$, a section of which is the deformation tensor $T$. As a result, we also distinguish seven classes of harmonic diffeomorphisms, and one of them, as was shown in [10, is described by Theorem 1.3.

\section{§3. INFINITESIMAL HARMONIC TRANSFORMATIONS OF RIEMANNIAN AND COMPLEX MANIFOLDS}

3.1. In a neighborhood $U$ of any point of a Riemannian manifold $(M, g)$, an arbitrary vector field $\xi \in C^{\infty} T M$ generates a local one-parameter group of infinitesimal transformations $\varphi_{t}: U \rightarrow M$ determined by the formulas

$$
\varphi_{t}(x)=\bar{x}^{k}+t \xi^{k},
$$

where $\left\{x^{1}, \ldots, x^{m}\right\}$ are local coordinates in $U, t \in(-\varepsilon,+\varepsilon) \subset \mathbf{R}$ is a parameter, and $\xi=\xi^{k} \partial_{k}$ (see [23, pp. 39-41] and [24, pp. 21-23]). For this reason, the vector field $\xi \in C^{\infty} T M$ is also called an infinitesimal transformation in $(M, g)$.

As a result of an infinitesimal transformation, the local components $g_{i j}$ of the metric tensor $g$ and the Christoffel symbols $\Gamma_{i j}^{k}$ take the following form:

$$
\bar{g}_{i j}=g_{i j}+t\left(L_{\xi} g_{i j}\right), \quad \bar{\Gamma}_{i j}^{k}=\Gamma_{i j}^{k}+t\left(L_{\xi} \Gamma_{i j}^{k}\right)
$$

(see [24, pp. 40, 41]). We have

$$
L_{\xi} g_{i j}=\nabla_{i} \xi_{j}+\nabla_{j} \xi_{i}, \quad L_{\xi} \Gamma_{i j}^{k}=\nabla_{i} \nabla_{j} \xi^{k}-R_{i j l}^{k} \xi^{l},
$$

and the components $R_{i j l}^{k}$ of the curvature tensor $R$ of the connection $\nabla$ are expressed in terms of the Lie derivatives of the components $g_{i j}$ of the tensor $g$ (see [23, p. 37]) and of the Christoffel symbols $\Gamma_{i j}^{k}$ with respect to the vector field $\xi$. 
Definition 3.1. A vector field $\xi$ is an infinitesimal harmonic transformation in $(M, g)$ if the local one-parameter group of infinitesimal transformations generated by $\xi$ in a neighborhood of any point of $(M, g)$ consists of infinitesimal harmonic transformations.

Applying formulas (3.1), we see that $\xi$ is an infinitesimal harmonic transformation if $\xi$ satisfies the equations

$$
g^{i j}\left(L_{\xi} \Gamma_{i j}^{k}\right)=0 .
$$

Theorem 3.1. A vector field $\xi \in C^{\infty} T M$ is an infinitesimal harmonic transformation in $(M, g)$ if and only if the components of $\xi$ satisfy the differential equations $\Delta \xi^{k}=2 R_{j}^{k} \xi^{j}$.

Proof. The Laplace-Beltrami operator $\Delta$ acts on an arbitrary vector field $\xi$ by the formula

$$
\Delta \xi^{k}=-g^{i j} \nabla_{i} \nabla_{j} \xi^{k}+R_{j}^{k} \xi^{j},
$$

where $R_{i j}=g_{i k} R_{j}^{k}$ are the components of the Ricci tensor Ric of $(M, g)$ (see [8, p. 203]). On the other hand, using (3.2), we can write equations (3.3) in the following equivalent form:

$$
g^{i j} \nabla_{i} \xi^{k}+R_{j}^{k} \xi^{j}=0 .
$$

These two systems of equations imply that $\Delta \xi^{k}=2 R_{j}^{k} \xi^{j}$.

Remark. In the case of Euclidean space, equations (3.6) turn into the system of LaplaceBeltrami equations $\Delta \xi^{k}=\sum_{j=1}^{m} \partial_{j}^{2} \xi^{k}=0$, whence the term "infinitesimal harmonic transformation".

\subsection{Examples of infinitesimal harmonic transformations.}

3.2.1. Our first example is an infinitesimal conformal transformation in $(M, g)$, i.e., a vector field $\xi$ satisfying the condition

$$
L_{\xi} g_{i j}=\nabla_{i} \xi_{j}+\nabla_{j} \xi_{j}=\frac{2}{m}\left(\nabla_{k} \xi^{k}\right) g_{i j}
$$

(see [21, p. 284]). The field $\xi$ is called an infinitesimal homothety if $\nabla_{k} \xi^{k}=$ const, and an infinitesimal isometry if $\nabla_{k} \xi^{k}=0$. The local one-parameter group of infinitesimal transformations generated by $\xi$ is conformal (homothetic or isometric) if and only if $\xi$ is an infinitesimal conformal transformation (an infinitesimal homothety or an infinitesimal isometry, respectively).

If $\xi$ is a conformal transformation, direct calculations show that

$$
\Delta \xi_{i}=2 R_{i j} \xi^{j}+m^{-1}(m-2) \nabla_{i}\left(\nabla_{k} \xi^{k}\right)
$$

(see, e.g., [5]). Therefore, each infinitesimal conformal transformation of a Riemannian 2manifold $(M, g)$ is harmonic. For other dimensions, this is possible only if the infinitesimal transformation in question is a homothety or an isometry.

By [5, Chapter III, Theorem 6.15], an infinitesimal conformal transformation of a compact Kähler manifold $(M, g, J)$ is an isometry, whence the following result.

Corollary 3.2. An infinitesimal conformal transformation of a compact Kähler manifold $(M, g, J)$ is harmonic. 
3.2.2. Suppose $(M, g, J)$ is a Kähler manifold. As our second example, we consider a vector field $\xi$ on $(M, g, J)$ that is an infinitesimal automorphism of the natural almost complex structure of $M$ (see [5, Chapter IV, §6]), i.e., $L_{\xi} J=0$. Since equations (3.4) are fulfilled on a compact Kähler manifold $(M, g, J)$ if and only if the vector field $\xi$ is an infinitesimal automorphism of the natural almost complex structure of $M$ (see [5, 7]), we obtain the following result.

Theorem 3.3. An infinitesimal automorphism of the natural almost complex structure of a compact Kähler manifold $(M, g, J)$ is a harmonic transformation. The converse is also true.

\subsection{Properties of infinitesimal harmonic transformations.}

Theorem 3.4. An infinitesimal harmonic transformation $\xi$ of a Riemannian manifold $(M, g)$ is isometric if $M$ is compact and the field $\xi$ is solenoidal.

Proof. We recall that the relations $\Delta \xi^{k}=2 R_{j}^{k} \xi^{j}$ and $\nabla_{j} \xi^{j}=0$ are fulfilled if and only if $\xi$ is an infinitesimal isometry of $(M, g)$ (see [8, p. 63]). It remains to use Theorem 3.1 .

Theorem 3.5. An infinitesimal harmonic transformation $\xi$ of a compact Kähler manifold $(M, g, J)$ of constant scalar curvature splits into a sum of the form $\xi=\xi^{\prime}+J \xi^{\prime \prime}$, where $\xi^{\prime}$ and $\xi^{\prime \prime}$ are infinitesimal isometries in $(M, g, J)$.

Proof. By Theorem 3.3, since $(M, g, J)$ is a compact Kähler manifold, an infinitesimal harmonic transformation $\xi$ of $(M, g, J)$ is an infinitesimal automorphism of the natural almost complex structure of $M$. On the other hand, by the A. Lichnerowicz theorem (see [5, Chapter IV, Theorem 8.1] and [8, p. 130]), since $(M, g, J)$ is a compact Kähler manifold of constant scalar curvature, each vector field $\xi$ as above on $(M, g, J)$ splits into a sum of the form $\xi=\xi^{\prime}+J \xi^{\prime \prime}$, where $\xi^{\prime}$ and $\xi^{\prime \prime}$ are infinitesimal isometries in $(M, g, J)$.

Theorem 3.6. A complete Riemannian manifold $(M, g)$ with negative Ricci curvature admits no nonzero infinitesimal harmonic transformations.

Proof. Applying the Laplace-Beltrami operator $\Delta$ to the function $F=g_{i j} \xi^{i} \xi^{j}$ for an arbitrary infinitesimal harmonic transformation $\xi$ of $(M, g)$, we obtain

$$
\Delta F=g^{i j} \nabla_{i} \nabla_{j}\left(g_{i j} \xi^{i} \xi^{j}\right)=2\left\lfloor-R_{i j} \xi^{i} \xi^{j}+g^{k l} g_{i j}\left(\nabla_{k} \xi^{i}\right)\left(\nabla_{l} \xi^{j}\right)\right\rfloor .
$$

Next, our assumption on the Ricci curvature of $M$ implies that $(M, g)$ is compact and $\Delta F \geq 0$. Finally, using the Hopf lemma (see [14, p. 308] and [24 p. 29]), we conclude that if (3.6) is fulfilled, then $\xi=0$.

\section{REFERENCES}

[1] J. Eells and L. Lemaire, A report on harmonic maps, Bull. London Math. Soc. 10 (1978), 1-68. MR.0495450 (82b:58033)

[2] , Another report on harmonic maps, Bull. London Math. Soc. 20 (1988), 385-584. MR.0956352 (89i:58027)

[3] İ. Davidov and A. G. Sergeev, Twistor spaces and harmonic maps, Uspekhi Mat. Nauk 48 (1993), no. 3, 3-96; English transl., Russian Math. Surveys 48 (1993), no. 3, 1-91. MR1243612 (95e:58046)

[4] L. P. Eisenhart, Riemannian geometry, Princeton Univ. Press, Princeton, 1949. MR0035081 $(11: 687 \mathrm{~g})$

[5] K. Yano, Differential geometry on complex and almost complex spaces, Internat. Ser. Monogr. in Pure Appl. Math., vol. 49A, Pergamon Press, New York, 1965. MR0187181 (32:4635)

[6] N. S. Sinyukov, Geodesic mappings of Riemannian spaces, "Nauka", Moscow, 1979. (Russian) MR.0552022 (81g:53014) 
[7] K. Yano, The theory of Lie derivatives and its applications, North-Holland, Amsterdam, 1957. MR.0088769 (19:576f)

[8] Sh. Kobayashi, Transformation groups in differential geometry, Ergeb. Math. Grenzgeb., vol. 70, Springer-Verlag, New York-Heidelberg, 1972. MF 0355886 (50:8360)

[9] H. Wu, The Bochner technique in differential geometry, Harvard Acad. Publ., London, 1987.

[10] S. E. Stepanov, The classification of harmonic diffeomorphisms, The 5-th International Conference on Geometry and Applications (August 24-29, 2001, Varna): Abstracts, Union of Bulgarian Mathematicians, Sofia, 2001, p. 55.

[11] J. Milnor, Morse theory, Ann. of Math. Stud., No. 51, Princeton Univ. Press, Princeton, NJ, 1963. MR.0163331 (29:634)

[12] S. E. Stepanov, On the global theory of some classes of mappings, Ann. Global Anal. Geom. 13 (1995), 239-249. MR1344481 (96k:58062)

[13] R. Narasimhan, Analysis on real and complex manifolds, Adv. Stud. Pure Math., vol. 1, Masson, Paris; North-Holland, Amsterdam, 1968. MR.0251745 (40:4972)

[14] Sh. Kobayashi and K. Nomizu, Foundations of differential geometry. Vol. 2, Intersci. Tracts in Pure Appl. Math., No. 15, Vol. II, John Wiley and Sons, Inc., New York etc., 1969. MR0238225|(38:6501)

[15] A. Gray and L. M. Hervella, The sixteen classes of almost Hermitian manifolds and their linear invariants, Ann. Math. Pura Appl. (4) 123 (1980), 35-58. MR0581924 (81m:53045)

[16] L. Friedland and Ch.-Ch. Hsiung, A certain class of almost Hermitian manifolds, Tensor (N.S.) 48 (1989), 252-263. MR1088471 (92b:53040)

[17] A. Gray, Nearly Kähler manifolds, J. Differential Geom. 4 (1970), 283-309. MR0267502 (42:2404)

[18] S. E. Stepanov, A group-theoretic approach to the study of Einstein and Maxwell equations, Teoret. Mat. Fiz. 111 (1997), no. 1, 32-43; English transl., Theoret. and Math. Phys. 111 (1997), no. 1, 419-427. MR1473424 (98k:83026)

[19] A. L. Besse, Einstein manifolds, Ergeb. Math. Grenzgeb. (3), vol. 10, Springer, Berlin etc., 1987. MR.0867684 (88f:53087)

[20] D. Kramer et al., Exact solutions of Einstein's field equations, Cambridge Univ. Press, Cambridge, 1980. MR0614593 (82h:83002)

[21] A. Nijenhuis, A note on first integrals of geodesics, Nederl. Akad. Wetensch. Proc. Ser. A 7029 (1967), no. 2, 141-145. MR0212697 (35:3563)

[22] S. E. Stepanov, On an application of a theorem of P. A. Shirokov in the Bochner technique, Izv. Vyssh. Uchebn. Zaved. Mat. 1996, no. 9, 53-59; English transl., Russian Math. (Iz. VUZ) 40 (1996), no. 9, 50-55 (1997). MR 1430472 (98b:53037)

[23] Sh. Kobayashi and K. Nomizu, Foundations of differential geometry. Vol. I, John Wiley and Sons, New York-London, 1963. MR0152974 (27:2945)

[24] K. Yano and S. Bochner, Curvature and Betti numbers, Ann. of Math. Stud., No. 32, Princeton Univ. Press, Princeton, NJ, 1953. MR0062505 (15:989f)

Vladimir State Pedagogical University, Pr. Stroitelei 11, Vladimir 600024, Russia

E-mail address: stepanov@vtsnnet.ru

Financial Academy, Government of the Russian Federation, Leningradskit Pr. 51, Moscow 125468, RUSSIA

E-mail address: igor-shandra@mtu-net.ru

Received 18/SEP/2001

Translated by N. YU. NETSVETAEV 